\title{
THE BASIS OF AREA AND DOT NUMBER EFFECTS IN RANDOM DOT MOTION PERCEPTION
}

\author{
Curtis L. Baker $J_{R}$ and Oliver J. BradDick \\ Departments of Physiology and Experimental Psychology, Cambridge University, \\ Downing Street. Cambridge CB2 3EG, England
}

(Received 21 October 1981; in revised form 16 April 1982)

\begin{abstract}
Random dot kinematograms present a patch of random dots, uniformly displaced from one frame to the next. We have re-examined the question of whether the limiting displacement for this short-range apparent motion is determined by the retinal angle or by the number of pixels across which the patch is displaced; our data support the former hypothesis. The number of dots in the patch is not a confounding variable, and in fact has very little effect. Increasing the area of the patch does increase the displacement limit, apparently due to the invasion of greater retinal eccentricities.
\end{abstract}

\section{INTRODUCTION}

The sensation of a smoothly moving textured planar surface can be elicited by "random dot kinematograms", in which a contiguous patch of randomly positioned dots is uniformly displaced from one frame to the next, in apparent motion (Anstis, 1970; Julesz, 1971). These stimuli are of great interest because analogously to random dot stereograms, they provide an instance of figure-ground segregation in which the only possible cue for segregation is the spatio-temporal relationship of successive frames.

That we can perceive motion in such patterns at all is of great interest, since it implies that the motion detection process, like stereopsis, is sophisticated enough to solve a "correspondence problem". That is, any individual dot in a particular frame will be followed by many random dots in nearby locations in the following frame, in addition to the dot in the location corresponding to the introduced displacement. Any process which simply responded to local dot pairings would signal many "false pairs", leading to perception of haphazard local motion. In order to make the correct correspondences only, the system must incorporate some "global" process, in the sense of a process which combines information from an extended set of dots. The analogous problem has been widely discussed in the case of stereopis (Julesz, 1971; Marr and Poggio, 1976). A particular algorithm for solving this "correspondence problem" in the case of motion has been suggested by Ullman, 1979).

Braddick (1974) demonstrated that the perceived segregation of a coherently moving region elicited by random dot kinematograms is mediated by a "short range process" which operates only for short interstimulus intervals (less than $100 \mathrm{msec}$ between successive frames) and small displacements (less than about $15 \mathrm{~min}$ arc). For displacements greater than this limit the motion-detecting process evidently becomes unable to establish the appropriate correspondence between successive frames, resulting in an impression of randomly-directed local motions. The processes responsible for apparent motion in classical stroboscopic displays, in contrast, operate over much longer interstimulus intervals and displacements (see Braddick (1980) for a review of the evidence for this dichotomy).

Lappin and Bell (1976) independently recognized that perception of random dot kinematograms is mediated by a process different in character from classical apparent motion. But, in contradiction to Braddick (1974), they presented evidence that the limiting displacement for correct motion perception was determined by the size of the displacement expressed as a number of array positions ("pixels"), rather than the retinal angle of the displacement.

These two views on the nature of the spatial limit carry rather different implications for the constraints dominating the underlying motion-detecting process. A fixed pixel limit would correspond to a limited number of possible false pairings of dots that could be handled; Lappin and Bell's (1976) cross-correlation model is essentially of this kind. A visual angle limit is more consistent with a process that only evaluates pairings over a fixed spatial range; such a range might be determined, for instance, by the dimensions of receptive field subunits at some point in the visual pathway.

The essential idea of both Braddick's (1974) and Lappin and Bell's (1976) experiments was to vary pixel spacing, and determine whether psychophysical performance was governed by the displacement, expressed as a number of pixels, which had not changed, or by the retinal angle of the displacement, which had changed. Lappin and Bell (1976) made this comparison for cases in which the size of the displaced patch, in pixels, was held constant (with a consequent co-varying change in retinal angle of the patch area). Braddick, however, chose to compare patches subtending equal retinal angles, with a conse- 
quent co-varying change in the number of pixels, and therefore the number of dots in his display. Lappin and Bell (1976) have argued that this change in the number of displaced dots was a confounding variable in Braddick's experiment. ${ }^{*}$

Some appreciation for this issue is gained by simply varying one's viewing distance from the display: the number of elements' displacement remains constant. but the retinal angle comprising that displacement decreases with viewing distance. For a display in which the displacement is slightly too great for perception of motion at a very close distance, backing away from the display can certainly provide an improvement in perceived motion, in contradiction to Lappin and Bell's hypothesis. But further increases in viewing distance do not give a further improvement in motion perceptibility, as would be expected if retinal angle alone were the determinant of the displacement limit. However, an increase in viewing distance also decreases the display area, a variable which has been reported to affect the displacement limit (Lappin and Bell, 1976; Chang and Juleš, 1981).

We shall show that the difference in findings is related to the means of equating area - the patch area, in retinal angle, has a marked effect on the displacement limit. We also examine the effects of varying dot density. This allows the number of false pairings within a given range to be viewed independently of pixel size. We find that this variation, with dot densities as low as $5 \%$, does not affect the displacement limit. In this situation pixel size is a parameter of pattern construction but is not evident from the pattern itself; a displacement limit in terms of pixels is therefore inapplicable. Furthermore, since changing dot density changes the number of dots in the pattern. this result implies that changes in dot number are not responsible for the area effect we find. Instead, evidence will be presented that the area effect can be accounted for by the increase in displacement limit with eccentricity.

\section{METHODS}

Each display consisted of two arrays of randomlypositioned dots, presented in succession on a computer-controlled display oscilloscope. Each frame was presented for $80 \mathrm{msec}$, with an interframe interval of $20 \mathrm{msec}$ determined by the refresh rate, giving a total stimulus duration of $160 \mathrm{msec}$. Each frame consisted of an $N \times N$ matrix of pixels, each of which was either empty or contained a dot. The second matrix

\footnotetext{
* Another difference between the two experiments was that Braddick (1974) scaled dot size along with pixel spacing while Lappin and Bell (1976) used dots of a constant size. However, Bell and Lappin (1973) scaled dot size and reached similar conclusions to their later paper on this point. In the present paper, dot size was not scaled except for the transitions between two viewing distances in the data of Fig. 7; it does not appear that dot size, in the range under consideration, makes a difference for the issue under discussion.
}

was identical to the first, except for a uniform displacement of dots within a central patch. The displacement was an integral number of pixels; a conventional "wrap-around" scheme was used, in which dots displaced beyond a patch boundary reappeared on the opposite side of the patch. Except as noted. dots occurred in each pixel with $50 \%$ probability. Viewing distances of either 1 or 2 metres were used. depending on the experiment (see below).

The spread of each individual dot on the screen was such that individual dots were just visibly distinct when they were spaced at $0.5 \mathrm{~mm}$ (pixel spacing in the experiments described was always at least twice this). A packed array of dots at this spacing had a luminance of $88 \mathrm{~cd} / \mathrm{m}^{2}$, which can thus be taken as an approximate estimate of the luminance of a dot. The dots appeared on a background luminance of $34 \mathrm{~cd} / \mathrm{m}^{2}$ from room illumination of the screen.

Other aspects of the display are described more fully in Baker and Braddick (1982).

Two types of psychophysical task were employed (Fig. 1).

(1) Segregation. The subject viewed a square array containing a rectangular patch of uniformly displaced dots; the surrounding dots of the array were uncorrelated in the two frames. Only one pair of frames was presented (total presentation time $160 \mathrm{msec}$ ). The percentage of errors in orientation discrimination (i.e. horizontal vs vertical rectangle) was scored.

(2) Direction discrimination. Again only one pair of frames was presented, but the subject's task was to discriminate the direction of motion (left or right) of the patch of uniformly displaced dots. The patch orientation was held constant, and there was no uncorrelated surround unless otherwise noted (in all the cases examined, the presence or absence of an uncorrelated surround had no effect on performance in this task).

Trials were preceded by a fixation mark, whose location indicated the centre of subsequent displays (except in experiments testing the effect of eccentricity). In all cases the subject initiated each trial by a button press. Discriminated conditions forientation or direction) were randomly chosen with equal probability. Following an initial task familiarization period. feedback as to correctness of responses was not provided to subjects. With the exception of experiments on the effect of area and eccentricity, all of the conditions being tested in each experiment were randomly interleaved.

Subjects consisted of one naive observer (R.M.C.) and five experienced psychophysical observers (ourselves, C.M.S., H.B.B. and P.P.).

\section{RESULTS}

Displacement limit determined by retinal angle, not pixels

In order to determine whether the decline of perceived motion with increasing displacement depends 
Segregation

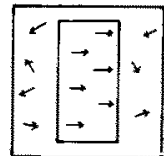

vs
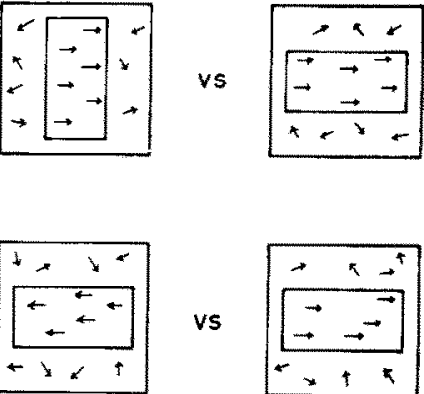

vs

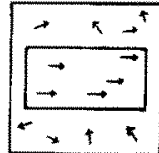

Fig. 1. Forced-choice psychophysical tasks used in our experiments. Top panels illustrate a segregation task, in which subject must judge the orientation (vertical vs horizontal) of a rectangular patch of uniformly displaced dots, which are embedded in a surround of randomly displaced dots. In the direction discrimination task (lower pancls), subject must report whether dots in the patch were displaced leftwards, or rightwards; in this case the surround pattern of uncorrelated dots is optional.

on the displacement expressed in retinal angle or in pixels, pixel spacings of $2.0,2.9$ and $4.7 \mathrm{~min}$ arc (viewing distance $2 \mathrm{~m}$ ) were used, with corresponding patch sizes of $20 \times 40,14 \times 28$, and $9 \times 17$ pixels respectively (thus giving a patch area of approx. $0.66 \times 1.33$ deg in all cases). The size of the uncorrelated surround was $50 \times 50$ pixels in all cases. Patch displacements of $1,2,3$ and 4 pixels were tested.

Figure 2 shows the results of this experiment for one subject, using both segregation and direction discrimination tasks. Note that in all cases the data points representing different pixel spacings show a very similar dependence on the displacement when expressed in retinal angle (left column), but not when expressed as number of elements or pixels (right column). Similar results were obtained on a second subject (P.P.).

These data confirm the conclusion reached by Braddick (1974) using a segregation response time task, and extend that result to other psychophysical tasks, including the direction discrimination used in studies by Lappin and Bell (1976). (The displacement limit here is smaller than that reported by Braddick (1974) because of the use of a smaller patch, as discussed under "effects of area")

A possible confounding variable in the experiment of Fig. 2 is the size of the surround, which varies with the pixel spacing. However, experiments in which the surround size alone was varied showed no significant effect of this variable.

A more cogent criticism of this experimental design, raised by Lappin and Bell (1976), is that the number of elements in the patch is varied reciprocally with pixel spacing (since retinal angle of the patch is held constant). They propose that the displacement limit increases with number of elements in the patch.

We sought to address this issue by repeating the experiment, but varying the dot density (percentage of pixels containing a dot) in a compensatory manner so

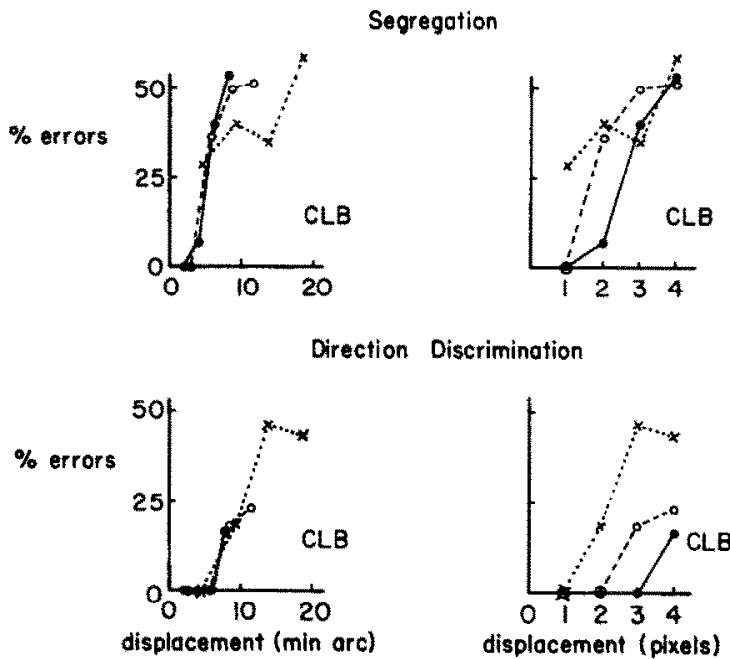

Fig. 2. Displacement in retinal angle, not number of pixels, determines performance in motion perception. Three pixel spacings were tested, with the patch area held at approx. $0.67 \times 1.34 \mathrm{deg} . \quad-$ patch $=20 \times 40$ pixels spaced at 2.0. $0-0:$ patch $=14 \times 28$ pixels spaced at $2.9^{\prime}$. $\times \ldots \ldots \times$ : patch $=9 \times 17$ pixels spaced at 4.6. Top panels show data for segregation task, lower panels for direction discrimination. Each data point represents percentage errors out of 60 trials.

as to maintain an approximately constant number of dots in the patch, for all pixel spacings. All aspects of the experiment, cxcept for dot density, were the same as in the previous experiment. Figure 3 illustrates examples of the displays used (only the rectangular patch is shown). (Dot size was constant and less than pixel spacing, so that, as illustrated, $50 \%$ density does not imply that $50 \%$ of the screen area was filled.)

a $20 \times 40,10 \%$

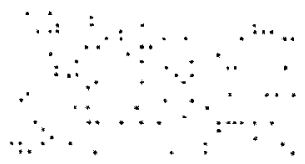

b

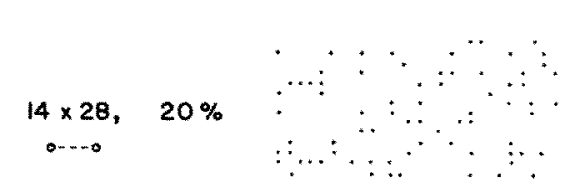

C

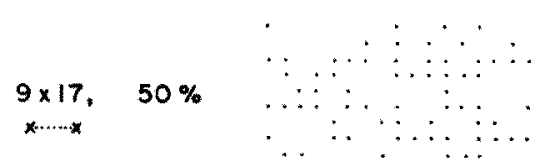

Fig. 3. Stimuli used to control against possible effects of number of dots, in experiment of Fig. 2. Dimensions of three random dot patches were chosen so as to subtend equal retinal angles, for three corresponding pixel spacings, as in experiment of Fig. 2. Dot densities were adjusted so as to maintain an approximately constant number of dots in each patch. Surround areas not shown. 
0

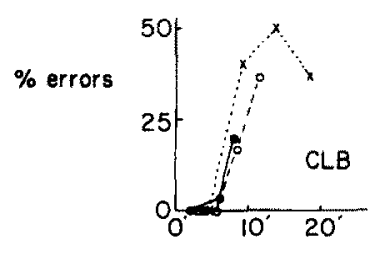

c

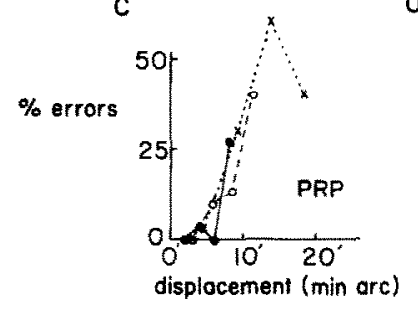

b
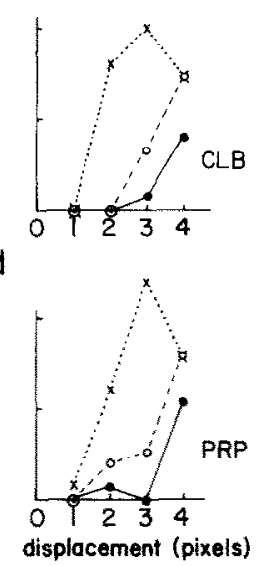

Fig. 4. Direction discrimination experiment as in lower panels of Fig. 2, except dot densities were changed so as to keep approximately the same number of dots in the test patch (see Fig. 3). - : patch $=20 \times 40$ pixels spaced at $2.0^{\prime} ; 10 \%$ dot density. $0-0:$ patch $=14 \times 28$ pixels, spaced at $2.9^{\prime}: 20 \%$ dot density. $\times \ldots \times$ : patch $=9 \times 17$ pixels, spaced at $4.6 ; 50 \%$ dot density. Each data point represents percentage errors out of 30 trials.

Figure 4 shows the results of this experiment for direction discrimination. Again. the data points for different pixel spacings plot almost superimposably when the displacement is taken as retinal angle, but not when represented as a number of elements. Thus Lappin and Bell's suggestion, that the apparent effect of pixel size results from changes in dot number, is not upheld.

\section{Dot density invariance}

The results shown in Figs 3 and 4 imply that the density of dots in the displaced patch has no effect on the displacement limit. This supposition was tested more fully by varying the dot density over a range of $1^{\circ} \%$ to $50 \%$ with a fixed pixel spacing.

Patches of $20 \times 40$ pixels, subtending approximately $0.77 \times 1.53$, were presented; the pixel spacing was $2.3^{\prime}$, at a screen distance of $2 \mathrm{~m}$. A direction discrimination task (no surround) used displacements of $2,3,4,5$ and 6 pixels, while a segregation task (uncorrelated $50 \times 50$ surround) used displacements of 1,2 , 3 and 4 pixels. Dot densities of 50,20,10, 5 and $1 \%$ were tested. Results of these experiments are shown in Fig. 5. Note that in all cases, dot density has very little effect on performance, except at the very lowest values. With the $1 \%$ density, segregation was harder for each value of displacement than with higher densities. This presumably reflects the poor definition of the patch boundary by very sparse dots; the effect is not present in the direction discrimination data.

In the low density cases, the row and column structure of the matrix is not discernible. Consequently. the pixel size is not available to the observer, and it is highly implausible that the performance of his visual

system is determined by displacement expressed in pixels.

\section{Effect of area}

To measure the effect of patch area on the displacement limit, a direction discrimination task was used with a patch of random dots of fixed size in pixels $(20 \times 20)$. Displacement was varied from 2 to 7 pixels. Element spacings of $1.15,2.3,3.45,4.60$ and $5.75 \mathrm{~min}$ arc (screen distance $2 \mathrm{~m}$ ) were used to obtain patch areas ranging from 0.15 to $3.67 \mathrm{deg}^{2}$. Values of displacement were randomly interleaved, but patch areas were not, because preliminary studies indicated that less variable results were obtainable when the subject had prior knowledge of the area of the forthcoming display. A block of 180 trials ( 30 repetitions for each of 6 displacements) was run for each pixel spacing, with a pseudorandom order of values.

In order to cover a larger range of areas, a further set of similar experiments was undertaken, using a $50 \times 50$ patch. with pixel spacings of 2.3. 4.6. 6.9.9.2 and $11.5 \mathrm{~min}$ arc (sercen distance $1 \mathrm{~m}$ ). providing patch areas ranging from 1.9 to $91.8 \mathrm{deg}^{2}$.

The results of these experiments are plotted in Fig. 6a. Data points connected by line segments correspond to the same patch area. Data for only a few values of patch area and displacements are shown. for clarity. Note that the percentage of errors increases with patch displacement more slowly as patch area increases. Similar results were also obtained on another subject (O.J.B.).

Figure $6 \mathrm{~b}$ illustrates a subset of the data from the above experiments, in which patch area is constant in terms of pixels (i.e. $50 \times 50$ ). Performance is plotted against displacement, also expressed in pixels, for fixed spacings of $2.3^{\prime}, 6.9^{\prime}$ and $11.5^{\prime}$. Notice that the

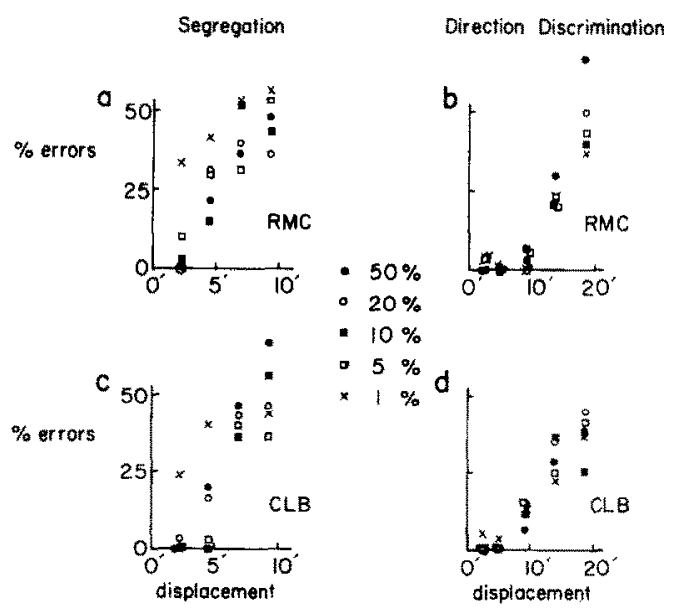

Fig. 5. Density of dots has surprisingly little effect on performance on either segregation $(a, c)$ or direction discrimination $(b, d)$. Only for a $1 \%$ density does segregation performance deteriorate, presumably because the patch boundary is poorly defined (average number of dots in patch $=8$ ). Each data point represents percentage errors out of 60 trials $(\mathrm{a}, \mathrm{d})$ or 30 trials $(\mathrm{b}, \mathrm{c})$. 


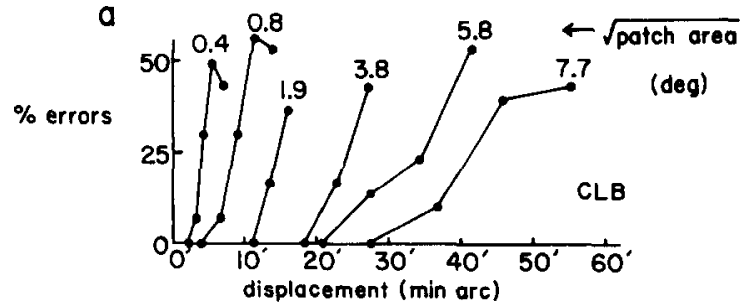

b

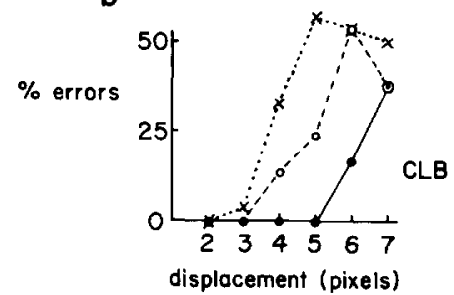

Fig. 6. Effect of patch area. (a) As the area of a moving patch of random dots is made larger, by increases in either pixel spacing or number of dots, the ability to perform correct direction discrimination improves dramatically. Results from only a few of the experiments in this series (see text) are illustrated, and several data points depicting error percentage of either $0 \%$ or ca. $50 \%$ are omitted, to preserve clarity of illustration. Data points connected by lines represent percentage errors out of 30 trials, for a patch size as indicated. (b) Failure of scaling invariance is shown when data from same experiment as above are plotted against displacement in pixcls. Data points connected by lines correspond to pixel spacings of $2.3^{\prime}, 6.9^{\prime}$ and $11.5^{\prime}$; in all of these cases, patch was $50 \times 50$ pixels. If scaling invariance (see Burt and Sperling. 1981) held, these three conditions should produce identical performance.

displacement limit, expressed in pixels, is clearly different for the different fixed spacings. Thus we fail to find the scaling invariance (i.e. independence of viewing distance, or equivalently, spatial scaling) observed by Lappin and Bell (1976), and by Burt and Sperling (1980) in a different type of experiment.

We used linear interpolation to estimate that value of displacennent which would have given a $20 \%$ error rate, for each value of patch area. This index of the displacement limit is plotted against the square root of patch area for two subjects in Fig. 7. Note that the data points deviate from a linear relationship for larger patch areas, indicating the failure of scaling invariance.

\section{Effect of eccentricity}

One plausible explanation of the above results would be that larger patch areas cover more peripheral regions of the retina, where the displacement limit may be greater. Pilot experiments testing this idea showed that the displacement limit for a large patch was not affected by occluding successively greater central portions of the patch, as long as a peripheral portion greater than some minimal area was visible.

Each subject was tested in a direction discrimination task with a large-area patch $(50 \times 50$ pixels

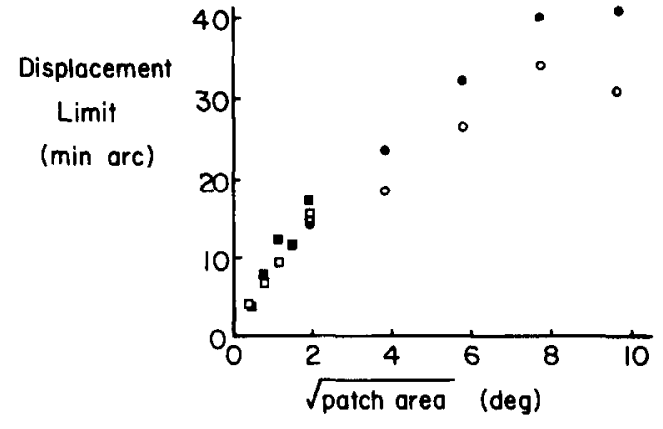

Fig. 7. Effect of patch area on displacement limit. Graphs of percentage errors as a function of displacement, as in Fig. 6a, were used to find interpolated displacement value corresponding to $20 \%$ errors; these values are plotted here, one for each patch size tested. Square symbols depict data tests using $20 \times 20$ pixel patches viewed at $2 \mathrm{~m}$, circles for $40 \times 40$ viewed at $1 \mathrm{~m}$. Note that the 2 deg point was replicated at each viewing distance, implying that the variation of dot size produced by changing the viewing distance was not a significant factor in this experiment. Solid symbols, C.L.B.; open symbols, O.J.B.

spaced at $9.2^{\prime}$, giving a $7.7 \times 7.7 \mathrm{deg}$ patch at $1 \mathrm{~m}$ ), whose center was offset from the fixation mark by 2.3 deg (right-most insert of Figs 8 and 9, data points shown as crosses connected by dotted line). In addition a series of smaller patches were used, each laterally offset from the fixation mark so as to lie just within the boundaries that would have been covered by the large area patch (middle inserts of Figs 8 and 9). Data are illustrated for only two of these small peripheral patches for each subject: the smallest one

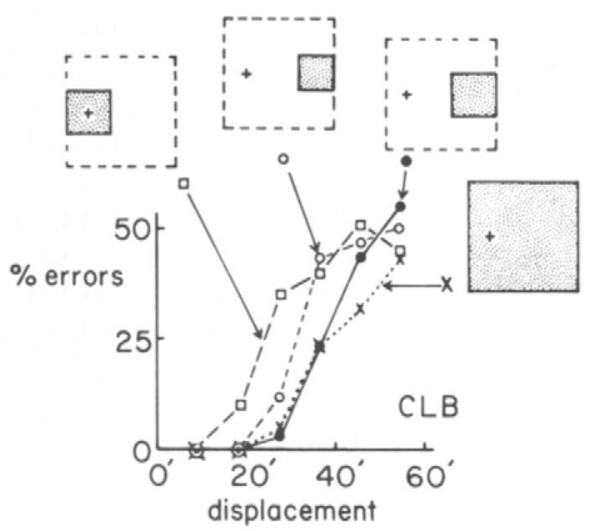

Fig. 8. Central vs peripheral contributions to the area effect. Graphs show percentage errors as a function of displacement in a direction discrimination task, for 4 patch configurations. Performance for a large-area patch (rightmost inset. data $\times \ldots \times)$ is identical to that for a small peripheral patch (-- Displacement limit is considerably less if the peripheral patch is too small $\left(\mathrm{O}_{---O}\right)$, or if it is foveally centred (left-most inset, $\square---\square$ ). Insets are drawn to scale: dashed squares indicate area that would be covered by large-area patch. Fixation mark indicated as + . Each data point represents percentage errors out of 60 trials. 


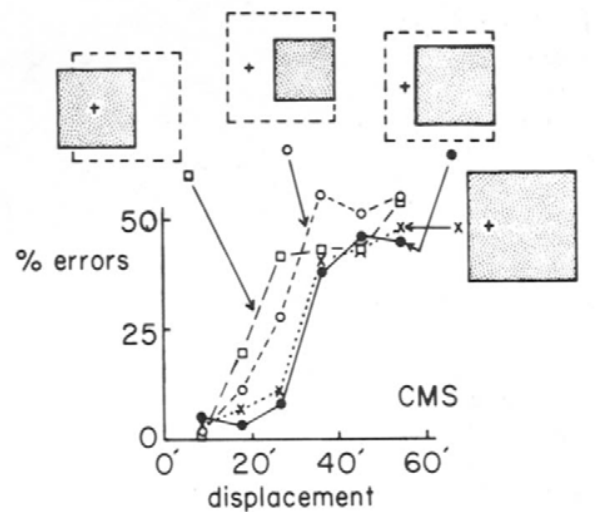

Fig. 9. Same as Fig. 8, for a subject who required a larger peripheral area in order to match performance with the large-area stimulus.

that had a displacement limit equal to that of the large-area stimulus (solid circles connected by dashed lines) and a slightly smaller one (open circles connected by dashed lines). For comparison, a small foveally-centered patch, equal in area to the former peripheral case, was also tested (left-most insert, data points shown as open squares connected by dashed lines).

Each of 6 displacements were tested in pseudorandom order, in 2 separate blocks of 180 trials (30 repetitions of 6 displacements) for each type of patch; these blocks of trials were themselves in pseudorandom order. In all cases the subject was instructed to maintain fixation on the location of the fixation mark.

Four subjects were tested in this manner (C.L.B., O.J.B., R.M.C., and C.S.M.). Figure 8 illustrates data for C.L.B., whose performance on the large-area patch was matched by the smallest area of peripheral patch $(3.1 \times 3.1 \mathrm{deg})$. Other subjects required larger peripheral areas to get displacement limits equal to that of the large-area stimulus, the greatest being $5.4 \times 5.4$ deg (C.M.S., Fig. 9).

We conclude that the improvement in displacement limit due to invasion of greater eccentricities is sufficient to explain the increase of displacement limit with area shown in Fig. 7: however, at a given eccentricity, the maximum value of displacement limit can be obtained only if the peripheral stimulus is larger than some critical size whose value varies across subjects.

We would emphasize that the range of eccentricities used in these experiments was quite restricted: it is quite possible that much greater displacement limits might be found in truly peripheral vision.

\section{DISCEISSION}

We have demonstrated that the displacement limit for perception of motion from random dot kinematograms is more consistently expressed as the retinal angle of that displacement, as first proposed by Braddick (1974), rather than the number of pixels, as advo- cated by Lappin and Bell (1976). Further, we have shown that the displacement limit increases with stimulus area. For increasing area centred at a parafoveal point, the displacement limit reached an apparent asympote for quite small areas (see Fig. 8); the steady increase found with larger areas covering the fovea (Fig. 7) is apparently due to invasion of the more eccentric parts of the visual field. These findings tend to support the notion that the displacement limit reflects physiological properties of the motion-detecting mechanisms.

The lack of effect of dot density seems to us the most important argument against displacement being limited by the number of shifted pixels. In the case of low density arrays (Fig. 3), a matrix-like structure (and, therefore, "pixels" as such) are no longer evident in the random dot pattern.

Lappin and Bell (1976) proposed that the number of dots in the patch was a critical variable, as predicted by a cross-correlation model of the neural processes underlying motion perception. But their experiment involved varying patch area concomitantly with the number of dots, and was therefore ambiguous.

To the extent that the dependence of displacement limit on the linear patch size (i.e., square root of area, see Fig. 7) can be approximated by a straight line through the origin, one can expect a reciprocity between displacement limit and pixel spacing for a patch of fixed number of pixels. Such a relationship would give a "scaling invariance" like that described by Burt and Sperling (1980). In our conditions such reciprocity seems to hold only for small patch sizes. For reasons as yet unknown, Lappin and Bell (1976) evidently obtained such reciprocity much more accurately (see especially their Fig. 3). One possible explanation for this discrepancy of findings may be the levels of contrast used: our displays were probably lower in contrast than those of Lappin and Bell, and thus may have recruited to a lesser degree motiondetectors in the periphery, where contrast sensitivity is reduced. This could cause our area effect curve (Fig. 7) to be lower for the larger areas.

A lack of effect of dot density on apparent motion has also been reported by Petersik (1980). using displays portraying a rotating sphere filled with random dots. In view of our conclusions, this result no longer serves as an argument that such 3-dimensional apparent motion is mediated by processes different from those underlying the 2-dimensional motion studied here.

Our finding that the displacement limit increases with eccentricity is reminiscent of the work by Foster et al. (1981), demonstrating (at greater eccentricities than those used here) that the spatial extent of this "fine-grain movement illusion" (FGMI) increases with eccentricity, in proportion to the cortical magnification factor. This and other similarities strengthen the liklihood that our short-range apparent motion and the FGMI reflect the operation of the same motion detection mechanism. 
It is not yet clear what is the absolute limit on displacement for the short-range motion process, or indeed, whether there is one. Blurring (low pass filtering), for example, extends the displacement limit, as can be easily observed by placing a diffusing screen (e.g. tracing paper) in front of a random dot kinematogram; Chang and Julesz (1981) have studied this effect quantitatively.

Acknowledgements-These results have been presented in abstract form (Baker and Braddick, 1981). We thank Horace B. Barlow, Rosalyn M. Cummings, Peter R. Packard and Caroline $M$. Stevenson for volunteering to act as subjects. Also, we are grateful to Horace Barlow and Suzanne McKee for many useful discussions, as well as their comments on the manuscript. This research was supported by a NATO Postdoctoral Fellowship to C.L.B., and an MRC grant to O.J.B.

\section{REFERENCES}

Anstis S. M. (1970) Phi movement as a subtraction process. Vision Res. 10, 1411-1430.

Baker C. L. Jr and Braddick O. J. (1982) Does segregation of differently moving areas depend on relative or absolute displacement? Vision Res. 22, 851-856.

Baker C. L. Ir and Braddick O. J. (1981) Random dot kinematograms: displacement limit depends on stimulus area. Soc. Neurosci. Abstr. 7, 81.8 .
Bell H. H. and Lappin J. S. (1973) Sufficient conditions for the discrimination of motion. Percept. Psychophys. 14, $45-50$.

Braddick O. J. (1974) A short-range process in apparent motion. Vision Res. 14, 519-527.

Braddick O. J. (1980) Low-level and high-level processes in apparent motion. Phil. Trans. R. Soc. Lond. B. 290 , 137-151.

Burt P. and Sperling G. (1981) Time, distance, and feature trade-offs in visual apparent motion. Psychol. Rev. 88. 171-195.

Chang J. J. and Julesz B. (1981) Disparity limits for s.f.filtered random dot kinematograms. Invest. Ophthal visual Sci. 20, 224.

Foster D. H., Thorson J., McIllwain J. T. and BiedermanThorson M. (1981) The fine-grain movement illusion: a perceptual probe of neuronal connectivity in the human visual system. Vision Res. 21, 1123-1128.

Julesz B. (1971) Foundations of Cyclopean Perception. Univ. of Chicago Press, Chicago.

Lappin J. S. and Bell H. H. (1976) The detection of coherence in moving random-dot patterns. Vision Res. 16, 161-168.

Marr D. and Poggio T. (1976) Cooperative computation of stereo disparity. Science 194, 283-287.

Petersik J. T. (1980) The effects of spatial and temporal factors on the perception of stroboscopic rotation simulations. Perception 9, 271-283.

Uliman S. (1979) in The Interpretation of Visual Motion. M.I.T. Press, Cambridge, M.A. 